\title{
Sweet Taste Perception in Pacific and NZ European Women is Associated with Dietary Intake and Eating

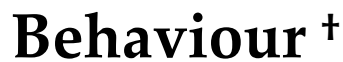

\author{
Sophie Kindleysides 1, Rozanne Kruger 1, Marine Corbin ${ }^{2}$, Marilize Richter ${ }^{1}$, Jeroen Douwes ${ }^{2}$ \\ and Bernhard H. Breier 1,3,* \\ 1 School of Sport, Exercise and Nutrition, College of Health, Massey University, Auckland 0745, \\ New Zealand; S.J.Kindleysides@massey.ac.nz (S.K.); r.kruger@massey.ac.nz (R.K.); \\ m.richter@massey.ac.nz (M.R.) \\ 2 Centre for Public Health Research (CPHR), College of Health, Massey University, Wellington 6140, \\ New Zealand; m.corbin@massey.ac.nz (M.C.); j.douwes@massey.ac.nz (J.D.) \\ 3 Riddet Institute Centre of Research Excellence, Palmerston North 4474, New Zealand \\ * Correspondence: b.breier@massey.ac.nz \\ + Presented at the 2018 Nutrition Society of New Zealand Annual Conference, Auckland, New Zealand, \\ 28-30 November 2018.
}

Published: 12 March 2019

Background: Taste perception may influence long-term dietary preferences, potentially contributing to the development of obesity. The aims of this study were to assess associations between sweet taste perception of glucose at suprathreshold concentrations with dietary intake and eating behaviour.

Methods: Sweet taste perception, assessed as intensity and hedonic liking, was measured in 304 women (18-45 years) selected on the basis of ethnicity (53\% NZ European and 47\% Pacific) and body fat percentage (BF\%; $51 \%$ normal and $49 \%$ high, using a cut-point of $35 \%$ ). Hierarchical cluster analysis was used to define sweet taste liking clusters. Dietary intake was assessed by 5 -day estimated food record, and eating behaviour was evaluated by the three-factor eating questionnaire (TFEQ). BF\% was measured by dual-energy x-ray absorptiometry (DEXA).

Results: Cluster analysis revealed that more (45\%) NZ European were sweet likers compared to Pacific (37\%), and more Pacific were sweet dislikers (63\%) compared to NZ European (55\%). NZ European sweet likers had a significantly higher intake of carbohydrates, sugars and starch $(199.4 \pm$ $51.1,87.9 \pm 27.4$ and $111.1 \pm 34.6 \mathrm{~g} /$ day $)$ when compared to sweet taste dislikers $(165.9 \pm 48.7,71.4 \pm$ 25.2 and $94.1 \pm 34.7 \mathrm{~g} /$ day, respectively; $p<0.001, p<0.001$, and $p<0.01$ ). In contrast, in Pacific women there was no difference in dietary intake between groups. Among normal BMI, sweet dislikers had a significantly higher intake of fat compared to sweet likers $(41 \%$ vs. $37 \%$ energy intake $p<0.01)$; conversely, sweet likers had a higher intake of carbohydrates $(42 \%)$ and sugar $(18 \%)$ in comparison to sweet dislikers ( $37 \%$ and $16 \%$ of energy intake, respectively; $p<0.05)$. Across all women, a higher emotional and situational dietary disinhibition score was associated with being a sweet liker $(p<0.05)$.

Conclusions: This study provides further evidence that sweet taste perception and hedonic liking of taste at suprathreshold levels are associated with dietary intake and eating behaviour. However, distinct differences may exist between population groups.

(C) 2019 by the authors. Licensee MDPI, Basel, Switzerland. This article is an open access article distributed under the terms and conditions of the Creative Commons Attribution (CC BY) license (http://creativecommons.org/licenses/by/4.0/). 